\title{
CARCASS CHARACTERISTICS AND MEAT QUALITY OF SAIDI LAMBS AS AFFECTED BY DIFFERENT BEDDING MATERIALS.
}

\author{
A.M.A. Hussein \\ Department of Animal Production, Faculty of Agriculture, Assuit University, Assuit, Egypt 71515
}

Received: 16/4/2019 Accepted: 7/5/2019

\section{SUMMARY}

The current study was carried out to study the effect of different bedding materials (sand, rice straw and wood shavings) on carcass characteristics, meat cuts and meat quality of Saidi lambs under upper Egypt condition. Eighteen lambs with $26 \pm 0.17 \mathrm{~kg}$ average body weight and approximately at 6 -months of age, fed ad. libitum with concentrate, were assigned to three groups of bedding in individual pens in replicates of six lambs each, for five months. Body measurements were taken once three days before slaughter using measuring tape and ruler. At the end of the experiment period (152 days), twelve lambs (4 from each treatment), were slaughtered, skinned and carcasses were prepared. Weight of non-carcass components, internal organs, fat stores, carcass parts and retail cuts were recorded. Semimembranosus (SM), longissimus dorsi (LD) and supraspinatus (SP) were dissected, weighted and used for meat chemical analyses and quality trats. Lambs reared on sand bedding had significant $(P<0.05)$ higher final body weight, round circumstances and pelvis width $P<0.05$. Moreover, lambs bedded with sand have higher $(P<0.05)$ liver weight and tests weight and kidneys fat weight $(P<0.05)$ compared with the other two groups. In addition, lambs in sand bedding gro up had significantly $P<0.01$ higher hot carcass weight, left side weight, right side weight, the fore carcass's quarters weights and dressing percentage compared with rice straw and wood shavings bedding groups. Also, lambs reared on sand bedding had significantly higher $(P<0.05)$ shoulder, brisket, rack, flank, loin, loin percent and round weight than those bedded with rice straw or wood shavings. It may be concluded that, sand bedding tends to improve meat chemical analysis and meat quality.

\section{Keywords:}

\section{INTRODUCTION}

In upper Egypt region, the high temperature in summer and low temperature in winter, enforce most of the lambs to be kept in covered or open-sided feedlot. Different types of the floor are used in those pens which vary from concrete, dirt or even sand. Mostly, sawdust, rice straw, wood shavings, and sand are the common beddings materials used in sheep industry (Hussein, 2018). Straw is an agricultural by-product and widely used as bedding materials (Ericsson and Nilsson, 2006) besides their role in providing warm, insulation and comfort (Tuyttens, 2005, Norring et al., 2010). The use of rice straw bedding materials may provide a source of feed which decrease the utilization of high concentrate diets (Fluharty et al., 1999) and consequently affect animals performance. On the other hand, sand is inorganic bedding material that provides comfort by conducting the heat away from animals body and its soft surface for laying (Bewley et al., 2001). In addition, sand has a suppressive effect on the bacterial population in bedding (Hogan et al., 1989, Bernard et al., 2003, Kristula et al., 2005). Consequently, by the suppression of bacterial growth, using sand as bedding materials may improve animals' health and performance. Although wood shavings is an organic material that can be used as bedding material for animals, it also like sand has a suppressive effect on bacterial population growth.
Moreover, wood shavings contain unique organic chemicals such as organic acids, resins, tannins, phenols, and turpentine which work as natural antibacterial (Allison and Anderson, 1951). Therefore, choosing the ideal bedding material depend on the balance between animal welfare and economic efficiency. Many studies have proved that different bedding material affected growing animal performance in terms of feed intake, feed conversion, body weight and daily gain (Wolf et al., 2010, Panivivat et al., 2004). Moreover, other studies found using different bedding materials can alter carcass characteristics and meat quality of animals (Teixeira et al., 2015). The aim of the current experiment is to compare the effect of three different bedding materials one of them is inorganic (sand) and two are organic materials (rice straw and wood shavings) on the carcass characteristics and meat quality of Egyptian Saidi lambs.

\section{MATERIALS AND METHODS}

\section{Animals and treatments:}

The animals and treatments are the same as shown in Hussein (2018). Briefly, eighteen Saidi male lambs with $26 \pm 0.17 \mathrm{~kg}$ average body weight and at approximately 6-months of age were fasted, weighed and blocked in descending order of weight. Lambs in each block were randomly allocated to one of the three treatment groups (rice straw, sand or 
wood shavings). Therefore, each treatment contained lambs that covered the full range of weights available, with six replicates each, in Randomized Complete Block Design (RCBD). Rice straw group was used as a control group, while the sand and wood shaving groups were investigated as alternative bedding materials. All lambs were housed in individual pens of $2.42 \mathrm{~m} 2$ bedded with one of the three bedding materials treatments. At the beginning, the bedding materials were initially laid to a depth of approximately $10 \mathrm{~cm}$ and then, were visually added weekly to each pen to maintain the desired depth of bedding materials. Moreover, the bedding materials in each pen were replaced with fresh one monthly (every 30 days). Wheat straw, concentrates mixture and water were supplied ad-libitum in buckets for each lamb. The ingredients of the concentrate mixture is as reported by Hussein (2018).

\section{Body measurements:}

Body measurements were taken once three days before slaughter using measuring tape and ruler. The body measurements were taken according to Yakubu (2013). Body measurements included, heart girth (HG), height at withers (HW), Chest depth (CD), body length (BL), Chest width (CW), Round circumference (RC), Paunch girth (PG), and Pelvis width (PW).

Slaughter, carcass characteristics and meat quality:

At the end of the experiment period (152 days), twelve lambs (4 from each treatment), were chosen according to their body weight to represent the mean final weight of each group. Before slaughter lambs were fasted from feeding for $14 \mathrm{~h}$ with free access to water. The lambs were weighed directly before and after slaughter.

The slaughtering process was done in the experimental abattoir by Islamic method of slaughtering animals (Halal). Where, by a very sharp knife a deep cut done instantaneously and quickly to the blood vessels of the neck (the two carotid arteries and the two jugular veins), the trachea and the esophagus but the central nervous system (the spinal cord) kept safe and intact. After slaughter, head, fore and hind feets were removed. Thereafter, the animals were skinned. Immediately, after skinning, each carcass was dressed, hot carcass and offal external components (skin, fore and hind feet and head) were weighed and recorded. Dressing percentage was calculated using hot carcass weight with respect to pre-slaughter live body weight. Moreover, internal organs include spleen, liver, lungs, heart, kidneys, rumen, intestine, and testes were weighed and recorded. Omental fat, mesenteric fat, kidney fat and pelvic fat were separated and weighed. Dressed carcass was separated into right and left sides, both sides were weighted. Carcass measurements were taken from the left side according to Abd-Alla (2014). Carcass measurements were carcass length, carcass depth at $3^{\text {th }}$ and $7^{\text {th }}$ rib, leg Length, leg circumference, loin length, and neck length.
Subsequently, both right and left sides were separated to fore and hind quarters at $13^{\text {th }}$ rib, the four quarters weights were recorded. Thereafter, each half of each carcass was separated into seven cuts as Strydom et al. (2009). These carcass cuts were loin, shoulder, rack (ribs), brisket, round, neck and flank. $9^{\text {th }}$ to $11^{\text {th }}$ ribs section was separated from rack in left side of the carcass following the procedure described by Morais et al. (2016). The section was weighted and separated to its components (muscle, fat and bone), thereafter the components were weighed and the percent of each component was calculated with respect to the whole section weight. Three major muscles, semimembranosus(SM), longissimus dorsi (LD) and supraspinatus (SP) were dissected and weighted. The three muscles were used as a sample for performing some meat quality testes $(\mathrm{pH}$, water holding capacity and cooking loose) and chemical analysis (moisture, dry matter, ash, protein and fat). The $\mathrm{pH}$ was measured by direct probe of $\mathrm{pH}$ meter (SCHOTT L 6880, Lab Star pH) applied on freshly cut surfaces of the three (LD, SM and SP) muscles. The meat samples from the three muscles LD, SM, and SP were weighed and cooked, in a water bath at $90 \mathrm{C}^{\circ}$, until the internal temperature reached $70 \mathrm{C}^{\circ}$ and left for one hour and weighed again to calculate cooking loss as described by Macit et al. (2003). Water holding capacity (WHC) was determined using filter paper pressing technique (Trout, 1988). For the chemical analysis, 150 grams minced meat were taken from each muscle for determining moisture, protein, ash and chemical fat following AOAC,2000 procedure.

\section{Statistical analysis:}

All collected data were analyzed using the Statistical Analyses System (SAS, 2013, version 9.4, SAS Institute Inc., Cary, NC, USA). The current study was designed as one-way RCBD with three treatments and sex blocks according to the following model for all data;

$$
y \hat{i j}=\mu+\mathcal{T} \hat{i}+\mathcal{B} \hat{j}+\boldsymbol{c} \hat{j}
$$

Where $y i j=$ the observation, $\mu=$ overall mean, $\mathcal{T} \hat{i}=$ effect of the $\mathrm{I}^{\text {th }}$ treatment, $\mathcal{B}_{\hat{j}}=$ effect of the $\mathcal{J}$ th block, $\varepsilon i j=$ the effect of the error related to individual observation. Before analysis normality test was proceeded by examination of normal distribution plots. All data passed the test and no data transformations were needed. comparis ons between the different treatments were done using the Duncan Multiple Range Test (Duncan, 1955).

\section{RESULTS.}

\section{Final body weight and pre-slaughtering body measurements:}

Final body weight and pre-slaughtering body measurements are shown in Table 1 . Lambs reared on sand bedding had significantly $(\mathrm{P}<0.05)$ higher final body weight, round circumstances and pelvis 
width compared with those reared-on rice straw or wood shavings beddings. On the other hand, there were no differences between the three bedding groups on the other body measurements (body length, height at withers, heart girth, Chest depth, Chest width, and Paunch girth). Moreover, both lambs bedded with rice straw and wood shavings have similar final body weight, round circumstances and pelvis width.

Table 1. Final body weight and body measurements of lambs as affected by bedding type

\begin{tabular}{lccc}
\hline & Sand & Rice straw & wood shavings \\
\hline Initial body weight (kg) & $26.42 \pm 1.01$ & $26.40 \pm 1.54$ & $26.40 \pm 1.17$ \\
Final body weight (kg). & $52.14 \pm 0.69^{\mathrm{a}}$ & $43.97 \pm 2.84^{\mathrm{b}}$ & $42.96 \pm 1.10^{\mathrm{b}}$ \\
body length (cm). & $73.01 \pm 0.11$ & $71.56 \pm 0.70$ & $71.76 \pm 0.33$ \\
height at withers (HW) & $72.84 \pm 0.09$ & $72.22 \pm 0.29$ & $72.25 \pm 0.14$ \\
heart girth (HG) & $89.32 \pm 0.35$ & $87.87 \pm 0.70$ & $87.80 \pm 0.21$ \\
Chest depth (CD) & $38.65 \pm 0.20$ & $38.03 \pm 0.31$ & $37.93 \pm 0.14$ \\
Chest width (CW) & $24.00 \pm 0.16$ & $23.38 \pm 0.31$ & $23.40 \pm 0.16$ \\
Round circumference (RC) & $43.03 \pm 0.09^{\mathrm{a}}$ & $42.43 \pm 0.24^{\mathrm{b}}$ & $42.38 \pm 0.13^{\mathrm{b}}$ \\
Paunch girth (PG) & $94.88 \pm 0.23$ & $92.70 \pm 1.20$ & $93.38 \pm 0.49$ \\
Pelvis width (PW) & $20.58 \pm 0.09^{\mathrm{a}}$ & $20.23 \pm 0.17^{\mathrm{ab}}$ & $20.18 \pm 0.06^{\mathrm{b}}$ \\
\hline
\end{tabular}

Different letters $(\mathrm{a}, \mathrm{b}, \mathrm{c})$ represent significant $(\mathrm{P}<0.05)$ differences among treatments.

\section{Slaughter by products, internal organs and fat stores:}

Head, feet and pelt weight are shown in Table 2. Head and feet weight did not affect by bedding type material. Moreover, the three groups had similar head and feet weight. In contrast, lambs bedded with sand had significant higher $(\mathrm{P}<0.05)$ belt weight compared with the other two groups, no difference in belt weight was found between lambs bedded with rice straw or wood shavings. Internal organs weight also, are affected by bedding material type (Table 2). In addition, lambs bedded with sand had higher internal organs weight compared with the other two groups. The differences were significant $(\mathrm{P}<0.05)$ only in case of liver weight and testes weight. On the other hand, kidneys weight was similar in the three groups. Rice straw group had remarkable significant $(\mathrm{P}<0.05)$ lower testes weight compared with the other two groups.

Table 2. Slaughter by products, internal organs and fat stores of lambs as affected by bedding type

\begin{tabular}{lccc}
\hline & Sand & Rice straw & wood shavings \\
\hline Head weight $(\mathrm{kg})$ & $2.91 \pm 0.16$ & $2.96 \pm 0.23$ & $2.81 \pm 0.28$ \\
Feet weight $(\mathrm{kg})$ & $1.05 \pm 0.06$ & $1.06 \pm 0.12$ & $1.01 \pm 0.06$ \\
Pelt weight $(\mathrm{kg})$ & $4.13 \pm 0.30^{\mathrm{a}}$ & $3.13 \pm 0.25^{\mathrm{b}}$ & $3.11 \pm 0.311^{\mathrm{b}}$ \\
Spleen weight $(\mathrm{g})$ & $69.26 \pm 9.81^{\mathrm{a}}$ & $62.57 \pm 8.84$ & $54.56 \pm 0.85$ \\
Liver weight $(\mathrm{g})$ & $657.15 \pm 40.77^{\mathrm{a}}$ & $486.41 \pm 27.36^{\mathrm{b}}$ & $427.85 \pm 18.69^{\mathrm{b}}$ \\
Lungs weight $(\mathrm{g})$ & $571.52 \pm 43.37$ & $552.37 \pm 63.71$ & $489.29 \pm 22.79$ \\
Heart weight $(\mathrm{g})$ & $241.36 \pm 19.73$ & $209.71 \pm 17.95$ & $204.12 \pm 3.98$ \\
Kidneys weight $(\mathrm{g})$ & $129.09 \pm 7.54$ & $129.29 \pm 20.92$ & $130.44 \pm 9.98$ \\
Rumen weight $(\mathrm{kg})$ & $1.53 \pm 0.16$ & $1.36 \pm 0.09$ & $1.42 \pm 0.12$ \\
Intestine weight $(\mathrm{kg})$ & $1.49 \pm 0.17$ & $1.33 \pm 0.10$ & $1.41 \pm 0.17$ \\
Testes weight $(\mathrm{g})$ & $445.80 \pm 56.86^{\mathrm{a}}$ & $298.18 \pm 32.72^{\mathrm{b}}$ & $418.56 \pm 51.38^{\mathrm{a}}$ \\
Kidneys fat weight $(\mathrm{g})$ & $147.22 \pm 28.09^{\mathrm{a}}$ & $64.55 \pm 12.56^{\mathrm{b}}$ & $87.31 \pm 27.24$ \\
Caul fat $(\mathrm{g})$ & $391.06 \pm 71.72$ & $155.99 \pm 53.05$ & $272.28 \pm 100.03$ \\
Omental fat $(\mathrm{g})$ & $268.40 \pm 77.37$ & $243.82 \pm 26.65$ & $270.67 \pm 38.82$ \\
Tail fat weight $(\mathrm{kg})$ & $3.32 \pm 0.77^{\mathrm{a}}$ & $2.35 \pm 0.58$ & $2.28 \pm 0.49$ \\
\hline
\end{tabular}

Different letters $(\mathrm{a}, \mathrm{b}, \mathrm{c})$ represent significant $(\mathrm{P}<0.05)$ differences among treatments.

Fat stores are represented in Table 2. Sand bedded lambs had higher kidneys fat, caul fat omental fat and tail fat than rice straw and wood shavings bedded lambs. In addition, rice straw bedded lambs had the lowest fats weight. While, wood shavings bedded lambs had intermediate fats weight. The only significant $(\mathrm{P}<0.05)$ value was in kidneys fat weight. In contrast, no significant differences in fat stores weight between lambs bedded with rice straw or wood shavings.

\section{Carcass weight, carcass parts weight and percent and carcass measurements:}

Hot carcass weight, dressing percentage, carcass parts and carcass measurements are shown in Table
3. Lambs in sand bedding group had significant $(\mathrm{P}<0.01)$ higher hot carcass weight, left side weight, right side weight, the fore carcass's quarters weights and dressing percentage compared with rice straw and wood shavings bedding groups. On the other hand, no differences were found between rice straw and wood shavings groups for hot carcass, dressing percentage or carcass parts. For carcass measurements only, internal carcass length and buttock circumference over tail were significantly $\mathrm{P}<0.05$ affected by bedding materials. Moreover, the sand bedded group had higher internal carcass length and buttock circumference over tail than rice straw bedding and wood shavings bedded groups. On the 
other hand, both rice straw and wood shavings buttock circumference over tail. groups had similar internal carcass length and

Table 3. Carcass weight, carcass parts weight and percent and carcass measurements of lambs as affected by bedding type

\begin{tabular}{|c|c|c|c|}
\hline & Sand & Rice straw & wood shavings \\
\hline Hot carcass weight $(\mathrm{kg})$. & $24.94 \pm 0.47^{\mathrm{a}}$ & $19.26 \pm 1.28^{b}$ & $18.40 \pm 0.63^{b}$ \\
\hline Dressing percentage $(\%)$ & $47.87 \pm 1.24^{\mathrm{a}}$ & $43.80 \pm 0.50^{b}$ & $42.80 \pm 0.39^{b}$ \\
\hline Right Side weight $(\mathrm{kg})$. & $12.27 \pm 0.31^{\mathrm{a}}$ & $9.37 \pm 0.54^{b}$ & $9.15 \pm 0.30^{b}$ \\
\hline Left Side weight $(\mathrm{kg})$. & $12.67 \pm 0.17^{\mathrm{a}}$ & $9.89 \pm 0.75^{\mathrm{b}}$ & $9.25 \pm 0.34^{\mathrm{b}}$ \\
\hline Left hind quarter weight $(\mathrm{kg})$. & $6.08 \pm 0.20^{\mathrm{a}}$ & $4.68 \pm 0.38^{b}$ & $4.30 \pm 0.11^{\mathrm{b}}$ \\
\hline Left fore quarter weight (kg). & $6.59 \pm 0.11^{\mathrm{a}}$ & $5.21 \pm 0.37^{\mathrm{b}}$ & $4.94 \pm 0.26^{\mathrm{b}}$ \\
\hline Right hind quarter weight ( $\mathrm{kg})$. & $5.86 \pm 0.25^{\mathrm{a}}$ & $4.41 \pm 0.28^{\mathrm{b}}$ & $4.25 \pm 0.10^{b}$ \\
\hline Right fore quarter weight $(\mathrm{kg})$. & $6.41 \pm 0.14^{\mathrm{a}}$ & $4.96 \pm 0.26^{\mathrm{b}}$ & $4.90 \pm 0.24^{\mathrm{b}}$ \\
\hline Right Side $\%$ & $49.19 \pm 0.33$ & $48.73 \pm 0.60$ & $49.74 \pm 0.28$ \\
\hline Left Side \%.). & $50.81 \pm 0.33$ & $51.27 \pm 0.60$ & $50.26 \pm 0.28$ \\
\hline Left hind quarter $\%$. & $24.35 \pm 0.48$ & $24.25 \pm 0.42$ & $23.43 \pm 0.59$ \\
\hline Left fore quarter $\%$. & $26.46 \pm 0.65$ & $27.07 \pm 0.26$ & $26.83 \pm 0.53$ \\
\hline Right hind quarter $\%$. & $23.48 \pm 0.63$ & $22.90 \pm 0.25$ & $23.15 \pm 0.49$ \\
\hline Right fore quarter $\%$. & $25.71 \pm 0.47$ & $25.83 \pm 0.44$ & $26.59 \pm 0.59$ \\
\hline Carcass length & $127.25 \pm 2.29$ & $121.75 \pm 2.15$ & $121.50 \pm 1.19$ \\
\hline Internal carcass length & $73.88 \pm 1.13^{\mathrm{a}}$ & $68.50 \pm 5.61^{b}$ & $67.63 \pm 1.55^{b}$ \\
\hline Carcass depth & $29.25 \pm 0.63$ & $28.25 \pm 0.97$ & $27.63 \pm 0.80$ \\
\hline Leg length & $41.25 \pm 1.31$ & $40.75 \pm 0.25$ & $40.75 \pm 1.11$ \\
\hline Leg circumference at $50 \%$ of length & $22.88 \pm 1.01$ & $23.50 \pm 0.29$ & $21.88 \pm 0.75$ \\
\hline Lumber region length & $24.50 \pm 0.94$ & $24.38 \pm 0.94$ & $24.75 \pm 0.88$ \\
\hline Neck length & $26.25 \pm 0.25$ & $24.50 \pm 0.79$ & $24.88 \pm 0.72$ \\
\hline Buttock circumference over tail & $46.50 \pm 2.01^{\mathrm{a}}$ & $43.00 \pm 1.59^{b}$ & $42.63 \pm 2.01^{b}$ \\
\hline Leg circumference undertail & $41.25 \pm 1.93$ & $41.63 \pm 1.60$ & $38.75 \pm 1.31$ \\
\hline
\end{tabular}
Different letters $(a, b, c)$ represent significant $(\mathrm{P}<0.05)$ differences among treatments.

Meat cuts and meat cuts percent:

Meat cuts and their relative percentages in respect to hot carcass are showed in Table 4. Lambs reared on sand bedding had significant higher $(\mathrm{P}<0.05)$ shoulder, brisket, rack, flank, loin and round weight than those bedded with rice straw or wood shavings. No significant differences were found between the rice straw and wood shavings in meat cuts weight. For the neck weight the only significant difference was between sand group and wood shavings group. The percent of each cut was calculated in respect to the left side and statistically analyzed. The significant difference $(\mathrm{P}<0.05)$ was only obtained in loin percent. Lambs bedded with sand had higher loin percent compare with wood shavings bedded lambs, no difference between the two other groups.

Table 4. Meat cuts and meat cuts percent of lambs as affected by bedding type

\begin{tabular}{lccc}
\hline Meat cuts & Sand & Straw & Wood shavings \\
\hline Shoulder weight $(\mathrm{kg})$. & $2.41 \pm 0.04^{\mathrm{a}}$ & $1.93 \pm 0.14^{\mathrm{b}}$ & $1.78 \pm 0.10^{\mathrm{b}}$ \\
Brisket weight $(\mathrm{kg})$. & $1.06 \pm 0.06^{\mathrm{a}}$ & $0.83 \pm 0.05^{\mathrm{b}}$ & $0.84 \pm 0.02^{\mathrm{b}}$ \\
Neck weight $(\mathrm{kg})$. & $1.29 \pm 0.05^{\mathrm{a}}$ & $1.05 \pm 0.10^{\mathrm{ab}}$ & $1.01 \pm 0.08^{\mathrm{b}}$ \\
Rack (rips) & $1.84 \pm 0.09^{\mathrm{a}}$ & $1.4 \pm 0.10^{\mathrm{b}}$ & $1.32 \pm 0.06^{\mathrm{b}}$ \\
Flank weight (kg). & $0.36 \pm 0.03^{\mathrm{a}}$ & $0.23 \pm 0.03^{\mathrm{b}}$ & $0.25 \pm 0.01^{\mathrm{b}}$ \\
Loin weight (kg). & $1.58 \pm 0.05^{\mathrm{a}}$ & $1.11 \pm 0.06^{\mathrm{b}}$ & $1.01 \pm 0.04^{\mathrm{b}}$ \\
Round weight (kg). & $4.13 \pm 0.14^{\mathrm{a}}$ & $3.35 \pm 0.32^{\mathrm{b}}$ & $3.05 \pm 0.09^{\mathrm{b}}$ \\
Shoulder \%. & $18.97 \pm 0.56$ & $19.54 \pm 0.19$ & $19.26 \pm 0.42$ \\
Brisket \%. & $8.38 \pm 0.35$ & $8.47 \pm 0.45$ & $9.08 \pm 0.11$ \\
Neck \%. & $10.20 \pm 0.53$ & $10.53 \pm 0.47$ & $10.87 \pm 0.50$ \\
Rack (ribs) \% & $14.50 \pm 0.66$ & $14.16 \pm 0.31$ & $14.18 \pm 0.33$ \\
Flank \%. & $2.87 \pm 0.17$ & $2.27 \pm 0.16$ & $2.68 \pm 0.20$ \\
Loin \%. & $12.47 \pm 0.38^{\mathrm{a}}$ & $11.27 \pm 0.53^{\mathrm{ab}}$ & $10.91 \pm 0.20^{\mathrm{b}}$ \\
Round \%. & $32.61 \pm 0.76$ & $33.76 \pm 0.71$ & $33.02 \pm 0.96$ \\
\hline
\end{tabular}

Different letters $(\mathrm{a}, \mathrm{b}, \mathrm{c})$ represent significant $(\mathrm{P}<0.05)$ differences among treatments.

Best rib weight, its component weight and percent and individual muscles weight:

Best rib is the carcass's cut of $9^{\text {th }}$ to $11^{\text {th }}$ rib. It is used as an indicator to meat, fat and bone percent in hall carcass. The best rib weight and its meat and fat was significantly $(\mathrm{P}<0.05)$ higher in lambs bedded with sand than those bedded with rice straw or wood shavings (Table 5). No differences were found in 
bone weight. Best neck components percent of meat and fat had a different response to the bedding material's type. Where, lambs bedded with sand had significant $(\mathrm{P}<0.05)$ lower meat percent and higher $(\mathrm{P}<0.05)$ fat percent than rice straw bedded lambs. In contrast, no differences were found between wood shavings and the other two groups in best neck components percent. Moreover, lambs bedded with sand had a significant higher $(\mathrm{P}<0.05)$ Supras pinatus, Semimembranosus and Longissimus dorsi muscles weight than the other two groups (Table 5). On the other hand, no differences were found in individual muscles weight between rice straw bedded lambs and wood shavings bedded lambs.

Table 5. Best rib weight, its component weight and percent and individual muscles weight of lambs as affected by bedding type

\begin{tabular}{lccc}
\hline & Sand & Rice straw & wood shavings \\
\hline Best Rib weight $(\mathrm{g})$. & $539.73 \pm 48.53^{\mathrm{a}}$ & $340.97 \pm 28.64^{\mathrm{b}}$ & $360.31 \pm 16.98^{\mathrm{b}}$ \\
Best Rib meat weight $(\mathrm{g})$. & $299.84 \pm 24.15^{\mathrm{a}}$ & $229.80 \pm 21.20^{\mathrm{b}}$ & $219.68 \pm 8.40^{\mathrm{b}}$ \\
Best Rib fat weight $(\mathrm{g})$. & $126.71 \pm 27.03^{\mathrm{a}}$ & $37.30 \pm 6.97^{\mathrm{b}}$ & $54.07 \pm 14.61^{\mathrm{b}}$ \\
Best Rib bone weight $(\mathrm{g})$. & $113.18 \pm 15.10^{\mathrm{a}}$ & $73.87 \pm 12.34$ & $86.56 \pm 9.37$ \\
Supraspinatus weight $(\mathrm{g})$. & $155.42 \pm 3.95^{\mathrm{a}}$ & $136.05 \pm 7.95^{\mathrm{b}}$ & $126.76 \pm 4.28^{\mathrm{b}}$ \\
Semimembranosus weight $(\mathrm{g})$. & $153.47 \pm 12.75^{\mathrm{a}}$ & $110.70 \pm 9.82^{\mathrm{b}}$ & $110.84 \pm 5.62^{\mathrm{b}}$ \\
Longis simus Dorsi weight $(\mathrm{g})$. & $108.00 \pm 16.40^{\mathrm{a}}$ & $85.26 \pm 11.93^{\mathrm{b}}$ & $83.01 \pm 1.83^{\mathrm{b}}$ \\
Best Rib meat \%. & $55.75 \pm 1.77^{\mathrm{b}}$ & $67.35 \pm 2.15^{\mathrm{a}}$ & $61.15 \pm 2.15^{\mathrm{ab}}$ \\
Best Rib fat \%. & $23.19 \pm 3.60^{\mathrm{a}}$ & $11.25 \pm 2.40^{\mathrm{b}}$ & $14.60 \pm 3.42^{\mathrm{ab}}$ \\
Best Rib bone \%. & $21.06 \pm 2.46^{\mathrm{b}}$ & $21.40 \pm 2.02$ & $24.25 \pm 3.04$ \\
\hline
\end{tabular}

Different letters $(\mathrm{a}, \mathrm{b}, \mathrm{c})$ represent significant $(\mathrm{P}<0.05)$ differences among treatments.

\section{Chemical analysis and meat quality treats:}

Meat chemical analysis, WHC, cooking loose and $\mathrm{pH}$ of LD, SM and SP muscles are shown in Table 6. Lambs bedded with sand tended to have lower moister content of their meat and consequently, higher DM in the different three muscles. The differences were significant $(\mathrm{P}<0.05)$ in case of $\mathrm{LD}$ muscle between sand bedded group and wood shavings group. One the other hand, the differences were not significant between the three groups in meat moisture and DM content in SM muscle. While, the chemical analysis of SP muscle showed a significant difference $(\mathrm{P}<0.05)$ between sand group and both rice straw and wood shavings group in moisture and DM percent. In addition, Lambs bedded with sand had significant $(\mathrm{P}<0.05)$ higher protein percent in the three individual muscles LD, SM and SP. For SP and $\mathrm{LD}$ muscles the significant difference was between sand group and the other two groups. However, in SM muscle both sand bedded group and rice straw bedded group had significant higher protein percent than wood shavings bedded group. The only significant difference in fat percent (Table 6) was found in SP muscle. Moreover, lambs bedded with sand had significant $(\mathrm{P}<0.05)$ higher fat percent than wood shavings group.

Table 6. Meat chemical analysis of lambs as affected by bedding type

\begin{tabular}{|c|c|c|c|c|c|c|c|c|c|}
\hline & \multicolumn{3}{|c|}{ Longissimus Dorsi muscle } & \multicolumn{3}{|c|}{ Semimembranosus muscle } & \multicolumn{3}{|c|}{ Supraspinatus muscle } \\
\hline & Sand & $\begin{array}{c}\text { Wood } \\
\text { shavings }\end{array}$ & $\begin{array}{l}\text { Rice } \\
\text { Straw }\end{array}$ & Sand & $\begin{array}{c}\text { Wood } \\
\text { shavings }\end{array}$ & $\begin{array}{l}\text { Rice } \\
\text { Straw }\end{array}$ & Sand & $\begin{array}{c}\text { Wood } \\
\text { shavings }\end{array}$ & $\begin{array}{l}\text { Rice } \\
\text { Straw }\end{array}$ \\
\hline Moisture \% & $\begin{array}{c}72.73 \pm \\
0.16^{\mathrm{b}}\end{array}$ & $\begin{array}{l}74.62 \pm \\
0.39^{a}\end{array}$ & $\begin{array}{c}73.06 \pm \\
0.77^{\mathrm{ab}}\end{array}$ & $\begin{array}{l}73.16 \pm \\
0.74\end{array}$ & $\begin{array}{l}75.47 \pm \\
1.20\end{array}$ & $\begin{array}{l}73.08 \pm \\
0.11\end{array}$ & $\begin{array}{c}71.83 \pm \\
0.45^{\mathrm{b}}\end{array}$ & $\begin{array}{c}75.81 \pm \\
0.66^{\mathrm{a}}\end{array}$ & $\begin{array}{l}74.34 \pm \\
0.36^{\mathrm{a}}\end{array}$ \\
\hline Dray Mater \%. & $\begin{array}{c}27.27 \pm \\
0.16^{\mathrm{a}}\end{array}$ & $\begin{array}{l}25.38 \pm \\
0.39^{\mathrm{b}}\end{array}$ & $\begin{array}{l}26.94 \pm \\
0.77^{a b}\end{array}$ & $\begin{array}{l}26.84 \pm \\
0.74\end{array}$ & $\begin{array}{c}24.53 \pm \\
1.20\end{array}$ & $\begin{array}{l}26.92 \pm \\
0.11\end{array}$ & $\begin{array}{l}28.17 \pm \\
0.45^{\mathrm{a}}\end{array}$ & $\begin{array}{c}24.19 \pm \\
0.66^{\mathrm{b}}\end{array}$ & $\begin{array}{c}25.66 \pm \\
0.36^{\mathrm{b}}\end{array}$ \\
\hline Protein $\%$. & $\begin{array}{c}21.93 \pm \\
0.41^{\mathrm{a}}\end{array}$ & $\begin{array}{c}20.17 \pm \\
0.48^{b}\end{array}$ & $\begin{array}{l}20.22 \pm \\
0.26^{\mathrm{b}}\end{array}$ & $\begin{array}{l}19.73 \pm \\
0.76^{\mathrm{a}}\end{array}$ & $\begin{array}{l}18.18 \pm \\
0.16^{b}\end{array}$ & $\begin{array}{l}20.02 \pm \\
0.10^{\mathrm{a}}\end{array}$ & $\begin{array}{c}19.84 \pm \\
0.42^{\mathrm{a}}\end{array}$ & $\begin{array}{c}18.30 \pm \\
0.58^{b}\end{array}$ & $\begin{array}{c}18.96 \pm \\
0.28^{\mathrm{ab}}\end{array}$ \\
\hline Fat $\%$. & $\begin{array}{l}3.87 \pm \\
0.45\end{array}$ & $\begin{array}{l}3.15 \pm \\
0.42\end{array}$ & $\begin{array}{c}4.70 \pm \\
0.52\end{array}$ & $\begin{array}{l}5.25 \pm \\
0.33\end{array}$ & $\begin{array}{l}4.01 \pm \\
0.95\end{array}$ & $\begin{array}{l}5.01 \pm \\
0.09\end{array}$ & $\begin{array}{l}6.67 \pm \\
0.65^{\mathrm{a}}\end{array}$ & $\begin{array}{l}4.19 \pm \\
0.37^{b}\end{array}$ & $\begin{array}{l}5.28 \pm \\
0.51^{\mathrm{ab}}\end{array}$ \\
\hline Ash \%. & $\begin{array}{c}0.98 \pm \\
0.02\end{array}$ & $\begin{array}{l}0.91 \pm \\
0.04\end{array}$ & $\begin{array}{l}1.00 \pm \\
0.05\end{array}$ & $\begin{array}{l}0.96 \pm \\
0.03\end{array}$ & $\begin{array}{l}0.95 \pm \\
0.02\end{array}$ & $\begin{array}{c}0.94 \pm \\
0.03\end{array}$ & $\begin{array}{c}0.89 \pm \\
0.02\end{array}$ & $\begin{array}{l}0.93 \pm \\
0.03\end{array}$ & $\begin{array}{c}0.92 \pm \\
0.01\end{array}$ \\
\hline Carbohydrate $\%$. & $\begin{array}{c}0.50 \pm \\
0.06^{\mathrm{b}}\end{array}$ & $\begin{array}{c}1.15 \pm \\
0.24^{\mathrm{a}}\end{array}$ & $\begin{array}{c}1.02 \pm \\
0.09^{\mathrm{a}}\end{array}$ & $\begin{array}{l}0.90 \pm \\
0.07^{\mathrm{b}}\end{array}$ & $\begin{array}{c}1.40 \pm \\
0.10^{\mathrm{a}}\end{array}$ & $\begin{array}{l}0.96 \pm \\
0.09^{\mathrm{b}}\end{array}$ & $\begin{array}{l}0.77 \pm \\
0.25\end{array}$ & $\begin{array}{c}0.78 \pm \\
0.22\end{array}$ & $\begin{array}{l}0.51 \pm \\
0.19\end{array}$ \\
\hline WHC \%. & $\begin{array}{l}42.22 \pm \\
1.80^{\mathrm{b}}\end{array}$ & $\begin{array}{l}48.59 \pm \\
0.78^{\mathrm{a}}\end{array}$ & $\begin{array}{l}40.73 \pm \\
0.58^{\mathrm{b}}\end{array}$ & $\begin{array}{l}42.20 \pm \\
2.39\end{array}$ & $\begin{array}{l}50.56 \pm \\
4.15\end{array}$ & $\begin{array}{l}46.96 \pm \\
1.74\end{array}$ & $\begin{array}{c}41.69 \pm \\
1.49\end{array}$ & $\begin{array}{l}46.00 \pm \\
2.55\end{array}$ & $\begin{array}{l}41.66 \pm \\
1.29\end{array}$ \\
\hline Cooking loss $\%$. & $\begin{array}{l}34.45 \pm \\
1.23 \mathrm{ab}\end{array}$ & $\begin{array}{l}35.77 \pm \\
0.90^{\mathrm{a}}\end{array}$ & $\begin{array}{c}32.10 \pm \\
0.62^{\mathrm{b}}\end{array}$ & $\begin{array}{l}38.13 \pm \\
0.13\end{array}$ & $\begin{array}{l}38.35 \pm \\
2.17\end{array}$ & $\begin{array}{c}38.77 \pm \\
0.49\end{array}$ & $\begin{array}{c}36.33 \pm \\
1.08\end{array}$ & $\begin{array}{c}34.46 \pm \\
1.52\end{array}$ & $\begin{array}{l}36.62 \pm \\
0.23\end{array}$ \\
\hline $\mathrm{pH}$ & $\begin{array}{l}5.72 \pm \\
0.04\end{array}$ & $\begin{array}{c}5.87 \pm \\
0.01\end{array}$ & $\begin{array}{l}5.71 \pm \\
0.03\end{array}$ & $\begin{array}{l}5.74 \pm \\
0.03\end{array}$ & $\begin{array}{l}5.82 \pm \\
0.04\end{array}$ & $\begin{array}{c}5.76 \pm \\
0.06\end{array}$ & $\begin{array}{l}5.75 \pm \\
0.06\end{array}$ & $\begin{array}{c}5.87 \pm \\
0.01\end{array}$ & $\begin{array}{c}5.73 \pm \\
0.04\end{array}$ \\
\hline
\end{tabular}

Different letters $(\mathrm{a}, \mathrm{b}, \mathrm{c})$ represent significant $(\mathrm{P}<0.05)$ differences among treatments. 
No significant differences were found among the three-experimental groups through the three analyzed muscles for ash percent. In contrast, lambs bedded with wood shavings had higher carbohydrates percent in the three analyzed muscles. For LD muscle, both wood shavings and rice straw groups had significant $(\mathrm{P}<0.05)$ higher carbohydrates percent than sand group. While, the carbohydrates percent was significant $(\mathrm{P}<0.05)$ higher in $\mathrm{SM}$ muscle for lambs bedded with wood shavings than those bedded with sand or rice straw. On the other hand, no significant differences were obtained between the three experimental groups in SP muscle for the carbohydrate percent. Water holding capacity, cooking loss and $\mathrm{pH}$ values as meat quality indicators are represents in Table 6 . The results showed that, lambs bedded with sand or rice straw had improvement in their meat WHC and cooking loss compared with those bedded with wood shavings. The improvement was only significant in case of $\mathrm{LD}$ muscle, no significant differences were recorded between sand bedded group and rice straw bedded group. $\mathrm{pH}$ values for three muscles showed no differences between the three experimental groups.

\section{DISCUSSION}

\section{Final body weight and body measurements:}

The final body weight results, had been discussed in the first published part of the current experiment (Hussein, 2018). Briefly, the dramatic increase of lambs' final body weight in sand group may be mainly due to two main reasons. Firstly, sand may be act through increasing lambs' laying time. Secondly, sand may enhance body weight by decreasing bacterial growth in bedding.

Many researches stated that sand bedding increases laying time(Cook, 2009, Teixeira et al., 2012). Because of increased laying times, several positive effects can be achieved on animal performance. Firstly, increased lying times increases rumination, which leads to enhance feed intake, improved digestion, and body weight (Robbins, 1983, Spalinger and C.T. Robbins, 1986, Van Soest, 1988, Maulfair et al., 2010). Secondly, long laying time influence the level of several hormones. The two important hormones cortisol and growth hormone level are positively correlated with laying time. High cortisol level is associated with longer laying time, which in turn has the ability to counteract the negative effects of stress on animals (Drissler et al., 2005). Moreover, higher level of growth hormone has been associated with long laying time and consequently, enhances body weight and general performance (Cook, 2009).

Sand bedding material has negative effect on bacterial growth and population (Kristula et al., 2005). Low bacterial population in animal's bedding improve animal health and performance (Hogan et al., 1989).

The higher body measurements of lambs reared on sand bedding may be due to higher body weight
(Table 1). Many authers showed that there are significant correlations between body weight and body measurements. Fore example, Younas et al. (2013) found a positive significant correlation between body weight of Hissardale sheep and all body measurements. Moreover, the authors reported that body measurement can be used for the estimation of body weight of animals when scales are not available. In addition, Abd-Alla (2014) found that fasted body weight of Barki lambs was positively correlated with paunch girth.

\section{Slaughter by products, internal organs and fat} stores:

The significant increase of belt weight, kidneys weight, tests weight and kidney fat weight as sociated with sand bedding is due to the higher body weight of lambs reared in sand bedding compared with those reard on rice straw or wood shavings beddings. Moreover, McCutcheon et al. (1993) found that there is a positive relationship between body weight and both non-carcass components and surface area in Romney rams. In the current study increased fat stores in lambs bedded with sand materials may be due to increased feed intake (Hussein, 2018). Moreover, Mahgoub et al. (2000) found that increased feed intake increases fat content of Omani lambs. In turn, higher carcass fat content of lambs reared in sand bedding can explain the higher dressing percentage of those lambs. Many authors (Karim et al., 2007, and Yalcintan et al., 2017) stated that higher fatness level in lambs consumed more feed and concomitant dressing percentage increase. In contrast to the current results, Day et al. (2006) and Wolf et al. (2010) reported that bedding type did not affect dressing percentage.

\section{Carcass weight, carcass parts weight and percent and carcass measurements:}

In the current study, the hot carcass weight and carcass parts weight even meat cuts weight differences reflect the body weight difference. In addition, lambs bedded with sand had higher final weight (table 1) so, their carcass weight, carcas s parts weight and meat cuts were higher than the other two groups. Many authors (Cankaya et al., 2009, Cam et al., 2010) found that there was a very strong relation between body weight and carcass weight. Although, there were significant differences in hot carcass weight, carcass parts and meat cuts, the carcass parts percent and meat cuts percent were not affected by different bedding materials. This indicates sand as bedding material can increase the carcass weight but cannot change carcass composition.

\section{Chemical analysis and meat quality treats:}

The improvement in chemical analysis and meat quality treats in the current study in sand group may be due to lambs comfort. In the published part of the current study (Hussein, 2018), it has been shown that lambs reared in sand bedding had significant lower serum cortisol concentration. Cortisol level affect 
carcass fat content, meatiness, and consequently carcass quality (Škrlep et al., 2009). Moreover, high cortisol level may affect post mortem proteolytic processes which in turn altered meat water holding capacity (WHC), flavor and tenderness (Ferguson and Warner, 2008). In contrast to our results, many authors (Ferguson and Warner, 2008, Teixeira et al., 2012) reported that bedding material did not affect carcas s quality.

\section{CONCLUSION}

The current study proved that sand as bedding material for lambs is better than both rice straw and wood shavings. In upper Egypt, sand is available, and it is very cheap material. Moreover, it is comfortable, and animals stay long periods laying down. Besides, it offers low bacterial load compared with organic materials. For all those reasons sand bedding improves lambs feed intake and body weight which in turn produce a heavier carcass with better meat quality.

\section{REFERENCES}

Abd-Alla, M. S. 2014. A comparative study on body measurements and carcass characteristics in Egyptian sheep and goats. Asian J. Anim. Vet. Adv 9:292-301.

Allison, F. E. and M.S. Anderson, 1951. The use of sawdust for mulches and soil improvement. U.S.D.A. Cir. 891.

Bernard, J. K., D. R. Bray, and J. W. West, 2003. Bacterial concentrations and sand usage in free stalls bedded with fresh or recycled sand. Pp. 001-008 in Fifth International Dairy Housing Proceedings of the 29-31 January 2003 Conference (Fort Worth, Texas USA).

Bewley, J., R.W. Palmer, and D.B. Jackson-Smith, 2001. A comparision of free-stall barns used by modernized Wisconsin dairies. J. Dairy Sci., 84:528-541.

Cam, M., M. Olfaz, and E. Soydan, 2010. Body measurements reflect body weights and carcass yields in Karayaka sheep. Asian Journal of Animal and Veterinary Advances 5(2):120-127.

Cankaya, S., A. Altop, M. Olfaz, and G. Erener, 2009. Canonical correlation analysis for estimation of relationships between some traits measured at pre-and post-slaughtering periods in Karayaka hoggets. Anadolu Tarm Bilimleri Dergisi 24(1):61-66.

Cook, N. B. 2009. Free stall Design for Maximum Cow Comfort. Pages 255 - 268 in Proc. Western Canadian Dairy Seminar Advances in Dairy Technology.

Day, J., T. Boland, and T. Crosby, 2006. The effects of plastic slatted floor or straw bedding on performance, liver weight and liver copper concentrations in intensively reared lambs. Livestock Science 100(2-3):270-275.
Drissler, M., M. Gaworski, C. B. Tucker, and D. M. Weary, 2005. Freestall maintenance: effects on lying behavior of dairy cattle. J. Dairy. Sci., 88:2381- 2387.

Ericsson, K. and J. L. Nilsson, 2006. Assessment of the potential biomass supply in Europe using a resource-focused approach. Biomass Bioenerg., 30:1-15.

Ferguson, D. and R. D. Warner, 2008. Have we underestimated the impact of pre-slaughter stress on meat quality in ruminants? Meat science 80(1):12-19.

Fluharty, F., G. Lowe, and D. Clevenger, 1999. Effects of pen floor type and bedding on lamb growth and carcass characteristics. SPECIAL CIRCULAR-OHIO RESEARCH AND AGRICULTURAL CENTER:107-111.

Hogan, J. S., K.L. Smith, K.H. Hoblet, D.A. Todhunter, P.S. Schoenberger, W.D. Hueston, D.E. Pritchard, G.L. Bowman, L.E. Heider, B.L. Brockett, and H.R. Conrad, 1989. Bacterial counts in bedding materials used on nine commercial dairies. J. Dairy Sci. 72(1):250-258.

Hussein, A. M. A., 2018. The physical characteristics of rice straw, wood shavings and sand as bedding materials and their effects on lambs' performance and welfare Egyptian J. Anim. Prod. 55(1):1-9.

Karim, S., K. Porwal, S. Kumar, and V. Singh, 2007. Carcass traits of Kheri lambs maintained on different system of feeding management. Meat Science 76(3):395-401.

Kristula, M. A., W. Rogers, J.S. Hogan, and M. Sabo, 2005. Comparison of bacteria populations in clean and recycled sand used for bedding in dairy facilities. J. Dairy. Sci., 88(12):4317-4325.

Macit, M., V. Aksakal, E. Emsen, N. Esenbuğa, and M. I. Aksu, 2003. Effects of vitamin E supplementation on fattening performance, noncarcass components and retail cut percentages, and meat quality traits of Awassi lambs. Meat Science 64(1):1-6.

Mahgoub, O., C. Lu, and R. Early, 2000. Effects of dietary energy density on feed intake, body weight gain and carcass chemical composition of Omani growing lambs. Small Ruminant Research 37(1-2):35-42.

Maulfair, D. D., G.I. Zanton, M. Fustini, and A.J. Heinrichs, 2010. Effect of feed sorting on chewing behavior, production, and rumen fermentation in lactating dairy cows. J. Dairy. Sci., 93:4791-4803.

McCutcheon, S., H. Blair, and R. Purchas, 1993. Body composition and organ weights in fleeceweight-selected and control Romney rams. New Zealand journal of agricultural research 36(4):445-449.

Morais, M. d. G., B. Biava de Menezes, C. Bertholini Ribeiro, C. C. Walker, H. J. Fernandes, A. R. Duarte Lopes Souza, C. C. Brandao Ferreira Itavo, and G. L. Dias Feijo, 2016. Models predict the proportion of bone, muscle, and fat in ewe 
lamb carcasses from in vivo measurements of the 9 th to 11th rib section and of the 12th rib. Semina: Ciências Agrárias 37(2).

Norring, M., E. Manninen, A. M. de Passillé, J. Rushen, and H. Saloniemi, 2010. Preferences of dairy cows for three stall surface materials with small amounts of bedding J. Dairy Sci., 93:70-74.

Panivivat, R., E.B. Kegley, J.A. Pennington, D.W. Kellogg, and S.L. Krumpelman, 2004. Growth Performance and Health of Dairy Calves Bedded with Different Types of Materials. J. Dairy Sci., 87(11):3736 - 3745.

Robbins, C. T. 1983, Digestion and Nutrient Metabolism. Pages 274-312 in Wildlife Feeding and Nutrition. Academic Press.

Škrlep, M., T. Kavar, V. Santé-Lhoutellier, and M. Čandek-Potokar, 2009. Effect of I199V polymorphism on PRKAG3 gene on carcass and meat quality traits in Slovenian commercial pigs. Journal of muscle foods 20(3):367-376.

Spalinger, D. E. and C.T. Robbins, 1986. The assessment of handling time in ruminants: the effect of plant chemical and physical structure on the rate of breakdown of plant particles in the rumen of mule deer and elk. Can. J. Zoolog., 64:312-321.

Strydom, P., L. Frylinck, J. Montgomery, and M. Smith, 2009. The comparis on of three $\beta$-agonists for growth performance, carcass characteristics and meat quality of feedlot cattle. Meat Science 81(3):557-564.

Teixeira, D. L., G. C. Miranda-de la Lama, M. Villarroel, S. Garcia-Belenguer, C. Sañudo, and G. A. María, 2012. Effect of straw on lamb welfare, production performance and meat quality during the finishing phase of fattening. Meat Sci., 92:829-836.

Teixeira, D. L., G. Miranda-de la Lama, M. Villarroel, J. L. Olleta, S. GarcÃa-Belenguer, J. Esc $\tilde{A}^{3} \mathrm{~s}$, and G. A. MarÃa, 2015. Effects of alternative bedding substrates on lamb welfare, productive performance, and meat quality during the finishing phase of fattening. J. Vet. Sci., 10(2):171-178.

Tuyttens, F. A. M., 2005. The importance of straw for pig and cattle welfare: A review. Appl. Anim. Behav. Sci., 92:261-282.

Van Soest, P., 1988. Nutritional ecology of the ruminant. Cornell Univ. Wood, GW:180-186.

Wolf, B. T., H.R.B. Molloy , M.J. Trayte, and M. T. Rose, 2010. Behaviour of growing lambs housed on straw or woodchip bedding materials and their preference for floor type Appl. Anim. Behav. Sci., 124:45-50.

Yakubu, A. 2,013. Principal component analysis of the conformation traits of Yankasa sheep. Biotechnology in Animal Husbandry 29(1):65-74.

Yalcintan, H., B. Ekiz, O. Kocak, N. Dogan, P. D. Akin, and A. Yilmaz, 2017. Carcass and meat quality characteristics of lambs reared in different seasons. Archives Animal Breeding 60(3):225233.

Younas, U., M. Abdullah, J. Bhatti, T. Pasha, N. Ahmad, M. Nasir, and A. Hussain, 2013. Interrelationship of body weight with linear body measurements in Hissardale sheep at different stages of life. J. Anim. Plant Sci 23(1):40-44.

\section{مواصفات الذبيحه وخصائص لحوم خر اف الصعيدي المرباه على أنواع مختلفة من الفرشه}

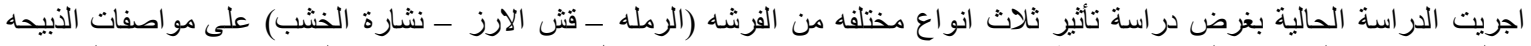

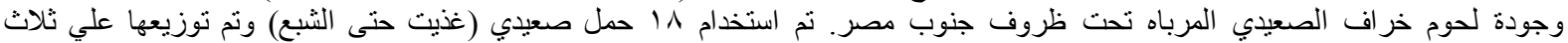

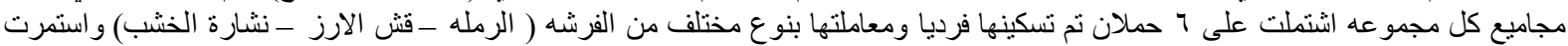

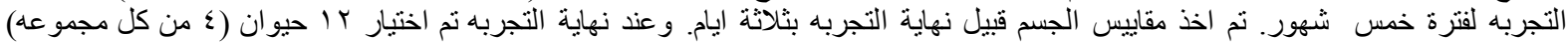

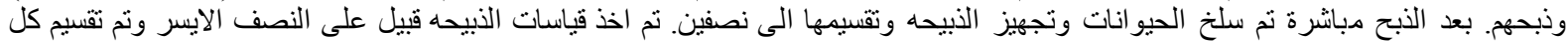

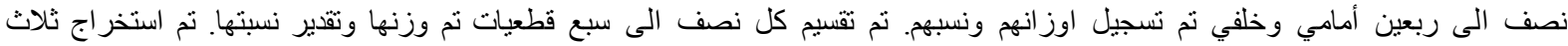

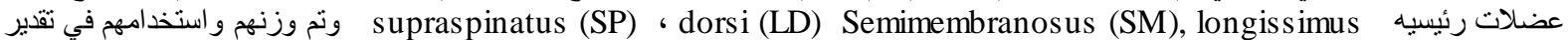

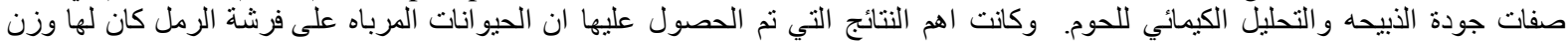

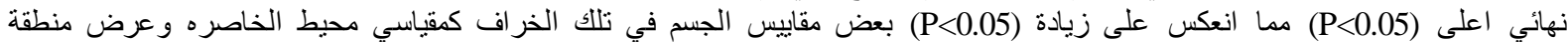

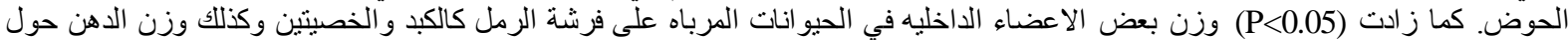

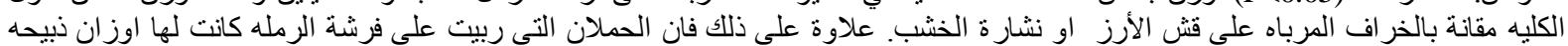

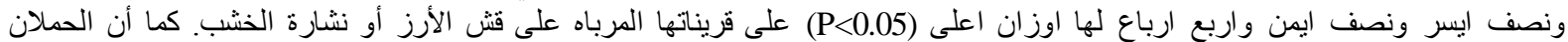

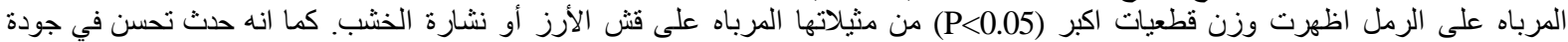

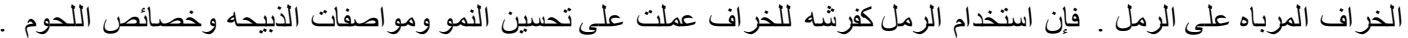

